\title{
Focused Ion Beam Prepared Cross-Sectional Transmission Electron Microscopy Preparation On CaGe2 On Ge(111) Grown By Molecular Beam Epitaxy
}

\author{
Robert E.A. Williams ${ }^{1}$, Jinsong Xuㄹ ${ }^{2}$, Amanda Hanks ${ }^{1}$, Adam Ahmed ${ }^{2}$, Igor V. Pinchuk ${ }^{2}$, Dave McComb ${ }^{1}$, \\ Roland Kawakami ${ }^{2}$, and Jyoti Katoch ${ }^{2}$ \\ 1. Center for Electron Microscopy and Microanalysis(CEMAS), The Ohio State University, Columbus, \\ Ohio 43212, United States \\ 2. Department of Physics, The Ohio State University, Columbus, Ohio 43210-1340, United States
}

Layered two-dimensional van der Waals materials exhibit uniquely tunable nanoscale properties with varying layer thickness and have attracted the attention of the scientific community due to their varied applications. Often these properties differ from the 3D bulk. For example, a single layer of graphite, graphene, has a linear dispersion relation and relativistic Dirac fermions, leading to the observation of many experimental phenomenon like atomic collapse, hofstadter's butterfly energy spectrum, etc, which remained elusive to researchers for many years. Transition metal dichalcogenides (TMDs) like $\mathrm{MoS}_{2}$ exhibit indirect to a direct band gap transition on going from bulk to a monolayer, resulting in potential applications in electronic, and optoelectronic devices. Direct experimental measurements by electron microscopy and other spectroscopy techniques on these materials is possible, in part, because of the weak in layer coupling, which permits exfoliation to obtain a monolayer or few layers. The ability to tune quantum properties kindles an interest to further explore and understand the novel properties of these layered 2D van der Waals materials with the dimensional confinement.

Since 2D materials are by definition a single-atom thick, they are rather sensitive to their environment and this provides a unique opportunity to further tune their properties by adatom doping and different ligand termination. For instance, graphene can be functionalized with hydrogen atoms to form graphane $(\mathrm{CH})$, which has a band gap or can be decorated with fluorine atoms to give insulating fluorographene $(\mathrm{CF})_{\mathrm{n}}$. Though adatom engineering does allow us to tune certain properties of graphene, it also results in an unwanted decrease in graphene's mobility and requires a long time for the adsorbed molecules to stabilize on its surface. This necessitates a need for low-dimensional materials beyond graphene, which have band gap and high spin orbit coupling for applications in nanoscale electronic and spin- based devices.

This need has led to an exploration into a new class of 2D van der Waals materials, which are group IV analogues (silicon, germanium and tin) of graphane. These materials have a hexagonal puckered lattice and are composed of $\mathrm{sp}^{3}$ hybridized group IV atoms, which are terminated with $\mathrm{H}$ or $(\mathrm{CH})_{n}$ ligands. This provides a versatile tool to tune the electronic properties of these $2 \mathrm{D}$ materials, such as high spin orbit coupling and band gap by different ligand termination, providing a method to tailor 2D materials for application in optoelectronic, sensing, thermoelectric and electronic devices as well as exploring exotic phenomena like spin Hall effect, and quantum spin Hall effect[1,2].

For realization of these quantum devices, it is necessary to produce uniformly large thin-films for engineering applications. However, the current state of development only allows for small regions of 2D material to be exfoliated, prohibiting the creation of viable commercial $2 \mathrm{D}$ devices. Recently, millimetersized bulk crystals of germanane $(\mathrm{GeH})$ and organic group-terminated germanane $\left(\mathrm{GeCH}_{3}, \mathrm{GeCH}_{2} \mathrm{CH}_{3}\right.$, $\mathrm{GeCH}_{2} \mathrm{CH}=\mathrm{CH}_{2}$ ) have been produced by topochemical deintercalation of layered zintl phase $\mathrm{CaGe}_{2}$ crystals in $\mathrm{HCl}$ and organoiodines respectively. It was observed that on replacing $\mathrm{H}$ with $\mathrm{CH}_{3}$ in $\mathrm{GeH}_{\text {the }}$ 
band gap increases by $0.1 \mathrm{eV}$ to $1.7 \mathrm{eV}$, as well as increasing the thermal stability from $75{ }^{\circ} \mathrm{C}(\mathrm{GeH})$ to 250 ${ }^{\circ} \mathrm{C}\left(\mathrm{GeCH}_{3}\right)$. Both $\mathrm{GeH}$ and $\mathrm{GeCH}_{3}$ can be exfoliated to obtain from few layers to a monolayer but their lateral sizes are only suitable for demonstration of prototype device physics, there is a need to fabricate large area thin films. In previous work, larger area germanane films having been synthesized by deintercalaction of $\mathrm{CaGe}_{2}$ layers grown by reactive deposition epitaxy (RDE) and we have reported molecular beam epitaxy (MBE) growth of $\mathrm{CaGe}_{2}$ on a $\mathrm{Ge}(111)$ substrate, followed by deintercalation.

In an attempt to improve the quality of the $\mathrm{CaGe}_{2}$ growth and therefore improve the resultant quality of deintercalation, an atomically flat Ge buffer layer was grown on $\mathrm{Ge}(111)$ to provide an ideal template for highly crystalline $\mathrm{CaGe}_{2} \mathrm{MBE}$ growth. Further exploration into deposition parameters were explored using a novel co-deposition technique for both Ge buffer layer growth, as well as $\mathrm{CaGe}_{2}$. To the author's knowledge, no previous research has imaged or shown direct observation of highly crystalline or epitaxial $\mathrm{CaGe}_{2}$ grown by any method. For this work, HR-STEM imaging was performed using a third-order probe corrected Titan $^{\text {TM }}$ STEM to provide feedback to growers and confirm the quality of growth. Images showing directly the quality of growth for both the buffer layer, as well as the $\mathrm{CaGe} 2$ layer were collected and filtered by correcting the nonlinear drift distortion using orthogonal scan pairs. [3]

\section{References:}

[1] Y. Kato, R. C. Myers, A. C. Gossard, and D. D. Awschalom, Nature 427 (6969), 50 (2004).

[2] Y. K. Kato, R. C. Myers, A. C. Gossard, and D. D. Awschalom, Science 306 (5703), 1910 (2004).

[3] Ophus, Colin, Jim Ciston, and Chris T. Nelson. Ultramicroscopy 162 (2016): 1-9.

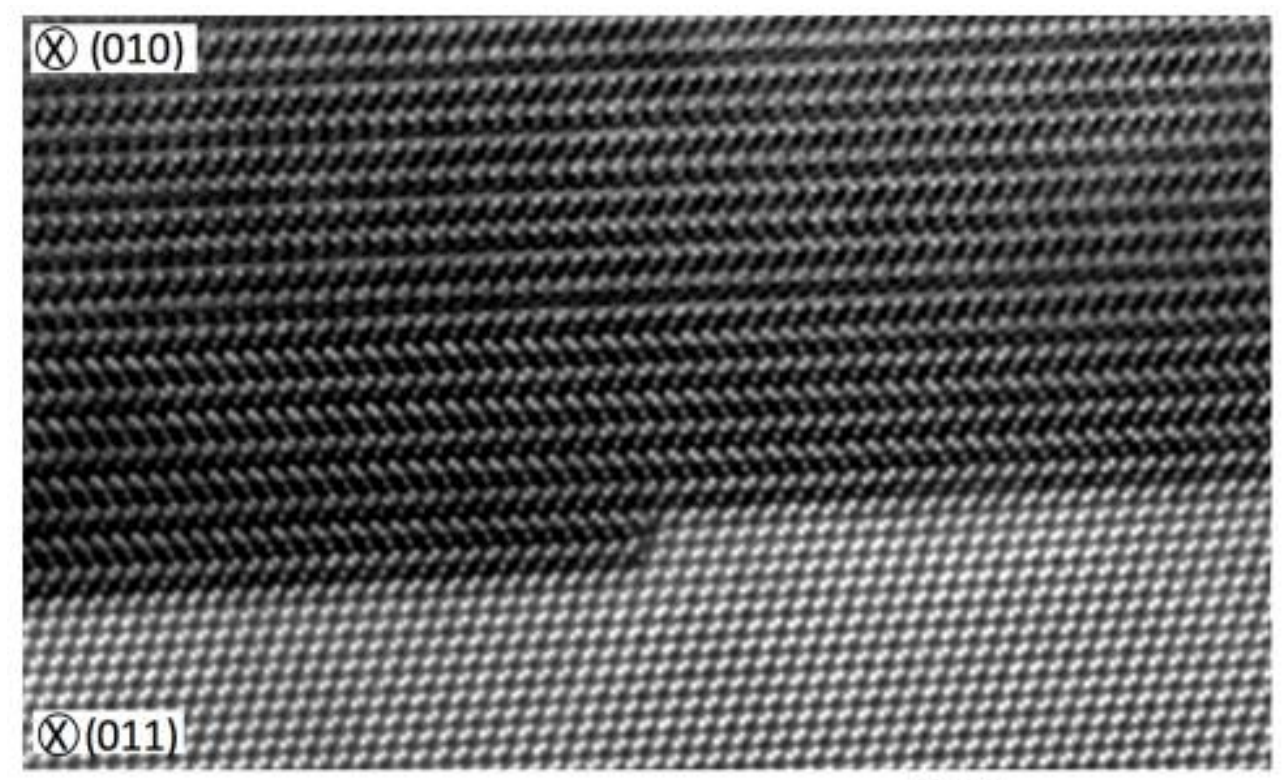

(111)


Figure 1: Probe corrected HR-STEM image, corrected for non-linear scan distortion. The bottom of the image is the MBE grown Ge layer, with the $\mathrm{CaGe}_{2}$ 